\title{
It is time to prepare for D-CARE!
}

\author{
Christoph Suppan, Marija Balic \\ Division of Oncology, Department of Internal Medicine, Medical University Graz, Graz, Austria \\ Correspondence to: Marija Balic. Division of Oncology, Department of Internal Medicine, Medical University Graz, Austria Auenbruggerplatz 15 , 8036 \\ Graz, Austria. Email: marija.balic@medunigraz.at. \\ Comment on: Coleman R, Finkelstein DM, Barrios C, et al. Adjuvant denosumab in early breast cancer (D-CARE): an international, multicentre, \\ randomised, controlled, phase 3 trial. Lancet Oncol 2020;21:60-72.
}

Submitted Apr 27, 2020. Accepted for publication May 06, 2020.

doi: 10.21037/atm-2020-99

View this article at: http://dx.doi.org/10.21037/atm-2020-99

The addition of antiresorptive agents in hormone-receptor positive breast cancer patients undergoing an adjuvant endocrine therapy with aromatase inhibitors is a common strategy to prevent treatment-induced bone loss. By suppressing the conversion from androgens to oestrogens, aromatase inhibitors lead to a decrease in oestrogen production resulting in a lower bone mineral density and increased fracture risk (1). Therefore, monitoring bone mineral density and-depending on the indicationinitiating treatment with bisphosphonates is recommended by most breast cancer treatment guidelines (2). In addition, treatment with bisphosphonates in postmenopausal patients with hormone-receptor positive breast cancer has been correlated to significantly lower fracture rates and lower recurrence rates and improved survival, whereas results have varied and the greatest benefits were seen in study-specific populations (1).

The largest cause of deaths related to cancer is, indeed, metastatic growth of disseminated tumor cells spread from the primary tumor. In light of these data, it is important to discuss molecular mechanisms in association with metastasis formation. One crucial step is the epithelial-tomesenchymal transition (EMT), when cancer cells cause upregulation of mesenchymal-associated genes that not only leads to loss of adhesion between cells, but also enables entry into the microvasculature of the blood and lymphatic system (3). From here, they pass through distant organs, leave the blood vessels and can settle down in another microenvironment. Studies in mouse models and analyses of clinical samples have implicated that dissemination of tumor cells in distant organs takes place very early in the process of tumorigenesis. Tumor cells cross the endothelial barrier and basement membrane of blood vessels in the bone marrow, thereby interacting with extracellular matrix components. Detected inside the bone marrow, these cells have been topic of long-term research and referred to as disseminated tumor cells (DTC). DTC are faced with several challenges in order to survive or proliferate. One important mechanism of cancer cells to survive is entering a quiescent state in order to wait for environmental changes that allow them to proliferate. This latency period is called tumor dormancy, where residual cancer is present but not clinically detectable. These dormant tumor cells may exist quiescently for many years or its proliferation is balanced by apoptotic cell death $(4,5)$.

Several studies on patients with operable breast cancer have investigated what value DTC in bone marrow have in prognosis, showing that about $12-45 \%$ of patients could have tumor cells in the bone marrow as determined by immunohistochemistry. In a pooled analysis of over 4,000 patients, the presence of micrometastases was detected in $30 \%$ and was associated with a poorer survival. However, the most common used method for detecting disseminated tumor cells was immunohistochemistry performed on bone marrow aspirates at early diagnosis. The procedure is invasive and does not represent additional burden for patients. While breast cancer metastases are hosted in bone marrow, it is still debated whether these cells are also metastatic progenitors for bone and non-bone metastasis. However, many studies support the idea of local growth of disseminated tumor cells into macrometastases (6).

A meta-analysis of patient data including over 18,000 breast cancer patients in adjuvant bisphosphonate randomized trials showed a reduction in breast cancer recurrences and breast 
cancer deaths. Adjuvant bisphosphonates included daily oral ibandronate, daily oral clodronate, or zoledronic acid. However, this effect was limited to postmenopausal women. Altogether, the decreased recurrence rates (RR 0.94, 95\% CI: $0.87-1.01 ; 2 \mathrm{p}=0.08)$, the distant recurrence rates $(0.92$, 95\% CI: $0.85-0.99 ; 2 \mathrm{p}=0.03)$ and breast cancer mortality (RR $0.91,95 \%$ CI: $0.83-0.99 ; 2 \mathrm{p}=0.04$ ) were defined as borderline significant. Bone recurrence was found to have decreased more significantly (RR 0.83 , 95\% CI: $0.73-$ 0.94; $2 \mathrm{p}=0.04)$. There was no effect on outcome seen in premenopausal women. In postmenopausal patients, there were highly significant reductions seen in the recurrence rate (RR 0.86, 95\% CI: $0.78-0.94 ; 2 \mathrm{p}=0.002$ ), in the distant recurrence rate (RR $0.82,95 \%$ CI: $0.74-0.92 ; 2 \mathrm{p}=0.0003$ ), the rate of bone recurrence (RR $0.72,95 \%$ CI: 0.6-0.86; $2 \mathrm{p}=0.0002)$ as well as the breast cancer mortality rate (RR $0.82,95 \%$ CI: $0.73-0.93 ; 2 \mathrm{p}=0.002)$. In addition, bone fractures were significantly reduced (RR $0.85,95 \%$ CI: $0.75-0.97 ; 2 \mathrm{p}=0.02)(1)$.

In ABCSG-12, premenopausal hormone-receptor positive breast cancer patients undergoing endocrine therapy and ovarian suppression did have the benefit of additional zoledronic acid every 6 months (7). However, this effect could not be confirmed in the AZURE trial, where, again, the subgroup of postmenopausal patients had improved outcomes (8).

Denosumab is a fully human monoclonal antibody which effectively and specifically binds to and inhibits the RANK ligand. The RANK-RANK-ligand system plays not only an important role in bone resorption and osteoclastogenesis, but might also have a suppressing effect in tumorigenesis (9). In terms of bone metastasis in solid tumors, denosumab is commonly used as a treatment to prevent skeletal-related events (10).

In light of the implementation of adjuvant bisphosphonates in international breast cancer treatment guidelines and its favorable toxicity profile, the denosumab's potential role in treating early breast cancer patients has been investigated for its efficacy on bone health and fracture risk, but also for its impact on disease specific outcomes.

ABCSG-18, a prospective, randomized, placebocontrolled, double-blind phase 3 trial, investigated adjuvant denosumab's effect on bone fractures along with other parameters of bone health, as well as on safety outcomes, in hormone-receptor positive postmenopausal breast cancer patients undergoing aromatase inhibitor therapy. In patients receiving adjuvant doses of denosumab at a rate of $60 \mathrm{mg}$ every six months, there was a significantly lower rate of fractures and the time until the first clinical fracture was significantly delayed (HR 0.5, 95\% CI: 0.39-0.65; $\mathrm{P}<0.0001)$ with no increase of denosumab-related toxic effects. Primarily aimed to prevent bone loss, an update of the study showed that disease-free survival in patients under added denosumab was significantly improved (HR 0.82, 95\% CI: 0.69-0.98, $\mathrm{P}=0.0260$ ). Disease-free survival in the denosumab group was $89.2 \%$ (95\% CI: 87.6-90.8) at 5 years, recorded at $80.6 \%$ (95\% CI: $78.1-83.1)$ after 8 years of follow-up versus $87.3 \%$ (95\% CI: $85.7-89.0$ ) at 5 years and $77.5 \%(95 \%$ CI: $74.8-80.2)$ at 8 years in the placebo group. Interestingly, no recorded cases of jaw osteonecrosis occurred $(11,12)$. However, the amount of both locoregional recurrences and contralateral recurrences, ductal carcinoma in situ or distant metastases were alike in both groups, and the effects on disease-free survival seem to be driven by new primary cancers (non-breast) and deaths without recurrences. Thus, the biologic effect of denosumab on disease-free survival remains partly unanswered.

D-CARE, on the other hand, is a randomized, placebocontrolled, double-blind phase 3 stud that assessed denosumab's effect in moderate to high-risk hormonereceptor positive and hormone-receptor negative early breast cancer patients on breast cancer outcomes as the primary objective. Denosumab was given at a dose of $120 \mathrm{mg}$ once every 28 days for 6 months, after which a 3 -month schedule followed over a total duration of 5 years. Bone-metastasis-free survival was defined as the primary endpoint and did not differ significantly between the two treatment groups (neither group reached the median; HR 0.97; 95\% CI: 0.82-1.14; $\mathrm{P}=0.70$ ). In addition, there was no significant effect of denosumab on disease-free survival or overall survival both in the overall population, and in the postmenopausal subgroup. In $5 \%$ of patients, positively adjudicated jaw osteonecrosis was reported under denosumab, compared to $<1 \%$ in the placebo group (13).

This trial failed to show any benefit of additional denosumab in the adjuvant setting while the ABCSG-18 trial clearly resulted in bone-protecting effects of denosumab at a lower dose. However, in light of these conflicting data, the direct anticancer effect of RANKL-inhibition still remains unclear. The most important differences between the two trials, which have so far investigated the role of denosumab in the early stage breast cancer, are primary objective (bone health/ fracture risk as primary objective of the ABCSG-18 vs. breast-cancer-specific outcome/ bone metastasis as the primary objective of D-CARE), dosing of denosumab, and differences in the study population. 
ABCSG-18 included only hormone-receptor-positive patients and the population was more homogenous than in the D-CARE trial, where up to $23 \%$ of patients were triplenegative. In the subanalyses there is a trend toward better outcomes in the hormone receptor positive patients with HR of 0.89 . Further important differences between both trials is the radiological follow-up. While D-CARE used an annual CT and bone scan surveillance programme, which is not standard practice, imaging in ABCSG-18 was driven by clinical symptoms. However, this might have had an effect on the primary endpoint, which was bone-metastasis-free survival, and it is not in line with international guidelines on follow-up of breast cancer patients in the adjuvant setting, considering the radiation exposure of a potentially curative patient population (14). Coleman et al. argues in an author's reply that results with robust endpoint monitoring, as it took place in the D-CARE trial, and 787 breast cancer recurrence cases may be more reliable than those which were generated by evaluating a secondary endpoint that followed analysis after the ABCSG-18 trial's early termination and unblinding, as the trial recorded 141 confirmed cases of breast cancer recurrence (15).

So what is the best available evidence now? It might have been easier if the comparator in ABCSG-18 had been a bisphosphonate-the standard of care in adjuvant breast cancer patients undergoing aromatase inhibitorsand not placebo. Notably, D-CARE investigated a higherrisk patient population, a larger sample size, had the same follow-up time and used a more intense dose of denosumab, without showing any benefit at the same time (16). The results of ABCSG-18 suggested that especially patients who started denosumab concomitantly with aromatase inhibitors within three months, had the greatest benefit from the treatment. On the other hand, in D-CARE, it was obligatory to start denosumab treatment within three months after surgery, so patients were in the perfect time window to receive the treatment $(11,13)$.

However, despite the promising effects of denosumab in this setting, there are still uncertainties concerning its benefit to survival, and data are contradictory.

Treatments that are able to modify the microenvironment and several interactions involved in the development of metastasis may also be able to change the course of the disease. Adjuvant bisphosphonates or denosumab both seem to play an important role in the metastatic process, which is not fully understood.

So far, we recommend continuing with the treatment of breast cancer patients under aromatase inhibitor therapy according to the international treatment guidelines, with the greatest evidence provided for bisphosphonates, both in terms of bone health and of disease outcome. We would, however, consider administering denosumab to selected patients reflecting the population of the ABCSG-18 trial, at lower doses and less frequently, since the benefit was associated with a low risk for patients. However, further data on long-term fractures should be awaited. D-CARE provided clear results that, for the overall population, there was no benefit seen when denosumab was added at the dose usually given for bone metastases.

\section{Acknowledgments}

Funding: None.

\section{Footnote}

Provenance and peer review: This article was commissioned by the editorial office, Annals of Translational Medicine. The article did not undergo external peer review.

Conflicts of Interest: Both authors have completed the ICMJE uniform disclosure form (available at http://dx.doi. org/10.21037/atm-2020-99). MB reports personal fees from Amgen, grants from Amgen, other from Amgen, grants, personal fees and other from Pfizer, from Roche, grants, personal fees and other from Eli Lilly, grants from Samsung, personal fees from Astra Zeneca, personal fees and other from Bayer, personal fees and other from MSD, outside the submitted work; CS reports personal fees from Pfizer, personal fees from Roche, personal fees from Novartis, personal fees from Lilly, personal fees from Astellas, personal fees from Pierre Fabre, outside the submitted work.

Ethical Statement: The authors are accountable for all aspects of the work in ensuring that questions related to the accuracy or integrity of any part of the work are appropriately investigated and resolved.

Open Access Statement: This is an Open Access article distributed in accordance with the Creative Commons Attribution-NonCommercial-NoDerivs 4.0 International License (CC BY-NC-ND 4.0), which permits the noncommercial replication and distribution of the article with the strict proviso that no changes or edits are made and the original work is properly cited (including links to both the 
formal publication through the relevant DOI and the license).

See: https://creativecommons.org/licenses/by-nc-nd/4.0/.

\section{References}

1. Early Breast Cancer Trialists' Collaborative Group (EBCTCG). Adjuvant bisphosphonate treatment in early breast cancer: meta-analyses of individual patient data from randomised trials. Lancet 2015;386:1353-61.

2. Hadji P, Aapro MS, Body JJ, et al. Management of aromatase inhibitor-associated bone loss in postmenopausal women with breast cancer: practical guidance for prevention and treatment. Ann Oncol 2011;22:2546-55.

3. Puisieux A, Brabletz T, Caramel J. Oncogenic roles of EMT-inducing transcription factors. Nat Cell Biol 2014;16:488-94.

4. Paez D, Labonte MJ, Bohanes P, et al. Cancer dormancy: a model of early dissemination and late cancer recurrence. Clin Cancer Res 2012;18:645-53.

5. Coleman R. Clinical benefits of bone targeted agents in early breast cancer. Breast 2019;48 Suppl 1:S92-6.

6. Vincent-Salomon A, Bidard FC, Pierga JY. Bone marrow micrometastasis in breast cancer: review of detection methods, prognostic impact and biological issues. J Clin Pathol 2008;61:570-6.

7. Gnant M, Mlineritsch B, Stoeger H, et al. Zoledronic acid combined with adjuvant endocrine therapy of tamoxifen versus anastrozol plus ovarian function suppression in premenopausal early breast cancer: final analysis of the Austrian Breast and Colorectal Cancer Study Group Trial 12. Ann Oncol 2015;26:313-20.

8. Coleman RE, Collinson M, Gregory W, et al. Benefits and

Cite this article as: Suppan C, Balic M. It is time to prepare for D-CARE! Ann Transl Med 2020;8(21):1339. doi: 10.21037/ atm-2020-99 risks of adjuvant treatment with zoledronic acid in stage II/III breast cancer. 10 years follow-up of the AZURE randomized clinical trial (BIG 01/04). J Bone Oncol 2018;13:123-35.

9. Lacey DL, Boyle WJ, Simonet WS, et al. Bench to bedside: elucidation of the OPG-RANK-RANKL pathway and the development of denosumab. Nat Rev Drug Discov 2012;11:401-19.

10. Van Poznak C, Somerfield MR, Moy B. Role of BoneModifying Agents in Metastatic Breast Cancer: An American Society of Clinical Oncology-Cancer Care Ontario Focused Guideline Update Summary. J Oncol Pract 2017;13:822-4.

11. Gnant M, Pfeiler G, Dubsky PC, et al. Adjuvant denosumab in breast cancer (ABCSG-18): a multicentre, randomised, double-blind, placebo-controlled trial. Lancet 2015;386:433-43.

12. Gnant M, Pfeiler G, Steger GG, et al. Adjuvant denosumab in postmenopausal patients with hormone receptor-positive breast cancer (ABCSG-18): disease-free survival results from a randomised, double-blind, placebocontrolled, phase 3 trial. Lancet Oncol 2019;20:339-51.

13. Coleman R, Finkelstein DM, Barrios C, et al. Adjuvant denosumab in early breast cancer (D-CARE): an international, multicentre, randomised, controlled, phase 3 trial. Lancet Oncol 2020;21:60-72.

14. Peters N, Conroy M, O'Reilly S. Adjuvant denosumab in early breast cancer. Lancet Oncol 2020;21:e121.

15. Coleman R, Zhou Y, Chan A. Adjuvant denosumab in early breast cancer - Authors' reply. Lancet Oncol 2020;21:e125.

16. Brandao M, Debiasi M, de Azambuja E. Denosumab in early-stage breast cancer. Lancet Oncol 2019;20:e234-5. 